\title{
Intensity Referencing and Response Linearization in an Optical-Reflection-Based Distance Sensor System for White-Goods Applications
}

\author{
Johannes Baumer ${ }^{1}$, Johann Schenkl ${ }^{1}$, Mikhail Shamonin ${ }^{2}$ \\ (mail address: Johannes.Baumer@emz-hanauer.de) \\ ${ }^{1}$ emz - Hanauer GmbH \& Co KGaA, Siemensstraße 1, D-92507 Nabburg, Germany \\ ${ }^{2}$ Laboratory for Sensor Technology, Regensburg University of Applied Sciences, \\ Postfach 1203 27, D-93025 Regensburg, Germany
}

\begin{abstract}
:
A reflective optical sensor system for distance measurements in white-goods applications is presented. The technical solution implements a well-known intensity referencing principle - the optical bridge - in a low-cost design. The influence of the ambient temperature can be compensated for. The spurious effects such as slow output drift caused by aging of optical components or the variation of the system sensitivity due to the parameter spreading of system components can be successfully overcome as well. An algorithm for linearizing the system response is described and optimized for a low-cost microcontroller. It is also shown how to calibrate the sensor system in a specifically developed automated assembly line prototype.
\end{abstract}

Key words: low-cost distance sensor, optical reflection, compensation of spurious effects, response linearization

\section{The challenge}

The trend is towards smaller washing machines with increasing loading levels. These two requirements contradict each other. The distance between the washing machine drum case and the outer case must be minimized, resulting in an increased risk of mechanical damage. The purpose of the proposed sensor system is to measure the relative position of the spin axis of the drum case in three dimensions (imbalance measurement).

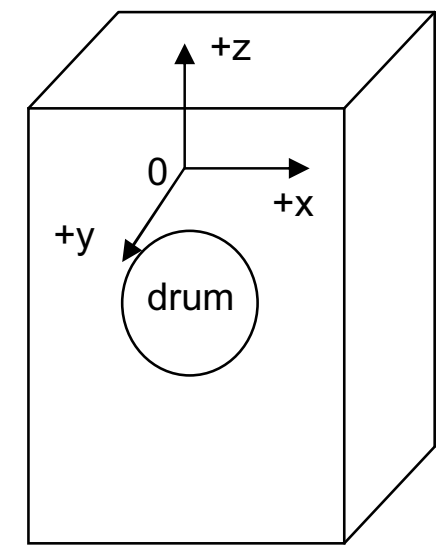

Fig.1. Sketch of a washing machine and the corresponding coordinate system

The second purpose of this system is to measure with high accuracy the absolute movement of the drum along the vertical axis ( $z$ - direction) during the loading process (see Fig.1.). It is done in order to obtain the weight of the loaded laundry. This information helps the designer of the machine to optimize the washing process or to easily display the load level (weight measurement).

An additional constraint is that such a system must be low cost in order to comply with the market requirements.

These tasks are solved by measuring the intensity of light reflected from the drum case or an additional reflector, which must be fixed to the drum case. Figure 2 shows the working principle of the measurement system for one particular axis using two pairs of optical elements (two emitters $E_{1}, E_{2}$ and two receivers $R_{1}, R_{2}$ ).

\section{The innovation of the solution}

Optical sensors based on intensity measurement require some form of intensity referencing to avoid errors arising from parasitic losses and environmental influences [1,2] The state of the art of intensity referencing methods is reviewed in [3]. It is commonly believed that known techniques of referencing such as balanced bridge, divided beam systems or twowavelength referencing are not suitable for lowcost applications because of their complexity. The proposed optical measurement system is 
based on the optical bridge principle (see Fig. 2.). Although the proposed optical sensor system is a low-cost design, it delivers excellent performance over system's life time.

Furthermore, the problem of linearization of the sensor response is solved. It will be shown that the linearization via an interpolating polynomial method [4] provides very good results. The mathematical operations required to solve both the above mentioned problems (referencing and linearization) are performed and optimized in order to be able to use a low-cost microcontroller.

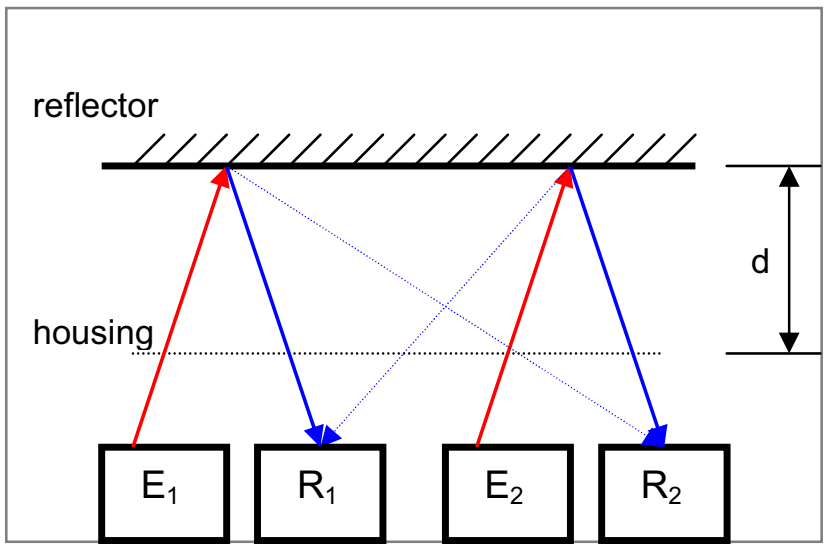

Fig.2. Distance measurement with an optical bridge side view ( $E$ designates an emitter, $R$ designates a receiver).

\section{Realization of the optical bridge}

Figure 3 shows the arrangement of the optical elements in the actual sensor system. The sensor system operates in a time-multiplexing mode. First, $E_{1}$ is switched on and the voltages at $R_{1}$ and $R_{2}$ are recorded (measurement signals $U_{11}$ and $U_{12}$, respectively). Next, $E_{1}$ is switched off, $E_{2}$ is switched on and the corresponding voltages $U_{21}$ at $R_{1}$ and $U_{22}$ at $R_{2}$ are measured. By building a contemporary quotient $Q_{\mathrm{OB}}$ (see Eq.1.), a compensated signal is generated.

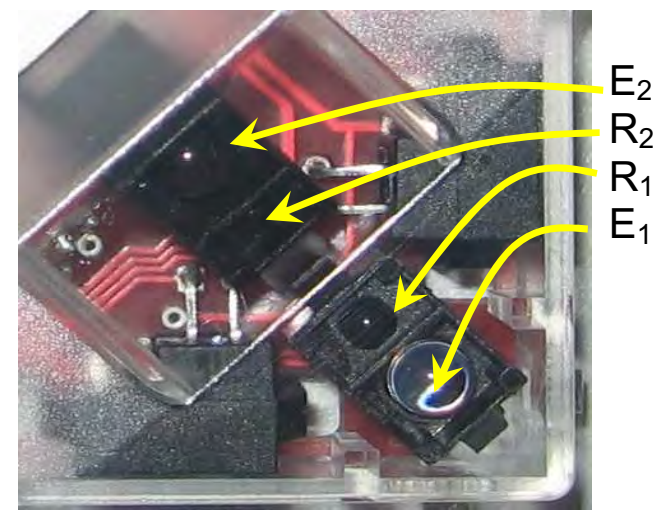

Fig.3. Picture of the realized arrangement of the optical bridge in a distance sensor
$Q_{O B}=\frac{U_{11} \bullet U_{22}}{U_{12} \bullet U_{21}}$

The specific arrangement of the optical elements results in a distance-dependent quotient $Q_{\mathrm{OB}}$, which tolerates spurious influences.

\section{Compensation for ambient temperature}

Figures 4 and 5 illustrate the improvement of the system performance with intensity referencing if the ambient temperature varies. Four sensors at a constant distance to a reflector were put into a temperature chamber where the temperature was raised from $10^{\circ} \mathrm{C}$ to $80^{\circ} \mathrm{C}$. The measurements using the voltage $U_{11}$ without intensity referencing resulted into a distance error of more than $3 \mathrm{~mm}$ (see Fig.4.).

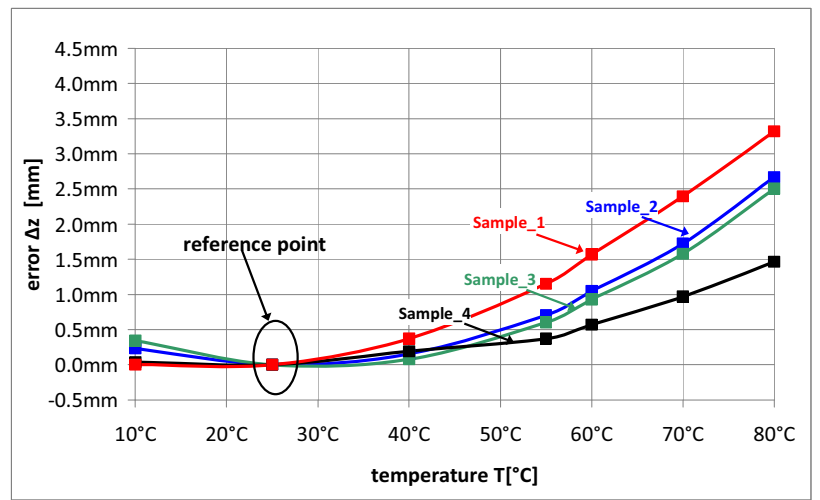

Fig.4. Error $\Delta z$ of distance measurement in $z-$ direction versus ambient temperature $T$ for four different sensors without intensity referencing.

Figure 5 shows the results of measurements with the same four sensors using the intensity referencing by the optical bridge. It is seen that the measurement error of $0.4 \mathrm{~mm}$ has been reduced almost by a factor of 10 .

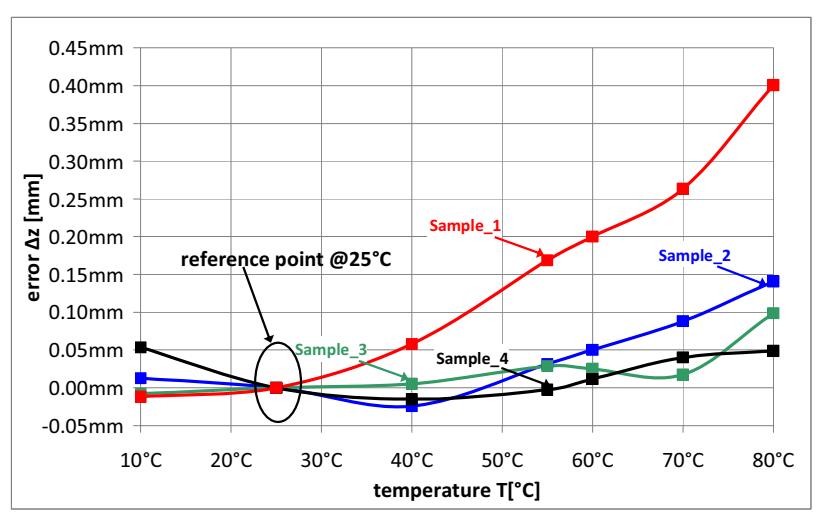

Fig.5. Error $\Delta z$ of distance measurement in $z$ direction versus ambient temperature $T$ for four different sensors with intensity referencing. 


\section{Compensation for effects of aging}

First, the slow drift of the system parameters is minimized by utilizing the quotient $Q_{\mathrm{OB}}$ if the optical components age in a similar way. This is achieved at the assembly line by using preselected similar elements for one sensor.

Second, the optical elements are operated in a pulsed mode. When the sensor system is in operation, the most-rapidly-aging elements - the emitters (IR - LEDs) - are effectively switched on during one tenth of the operation time.

The estimated life time of the sensor system is about 30000 hours before the performance deterioration due to the effects of aging may come into play.

\section{Compensation for variation of system elements}

The used low-cost elements show large spreading of their parameters. For example, the sensitivity of a pair of an emitter and a receiver may differ by a factor of five. Figure 6 presents the voltages $U_{i j}(i, j=1$ or 2 ) for one particular realization of a sensor system. It is seen that these voltages show different dependencies on the distance $z$. On the one hand, this different behavior of the four emitter-receiver pairs is caused by the asymmetrical design of the optical bridge. On the other hand, the variation of the element parameters (e.g. sensitivity) and their relative orientations contribute to the variation of the overall system characteristics as well. As a result, the quotient $Q_{O B}$ will not remain the same for each particular realization of the sensor system. So each particular sensor needs to be calibrated at the beginning of its operation life time. After this the sensor performance remains stable with respect to further small variations of system elements (caused e.g. by displacements due to the mechanical impact) because of the opticalbridge configuration.

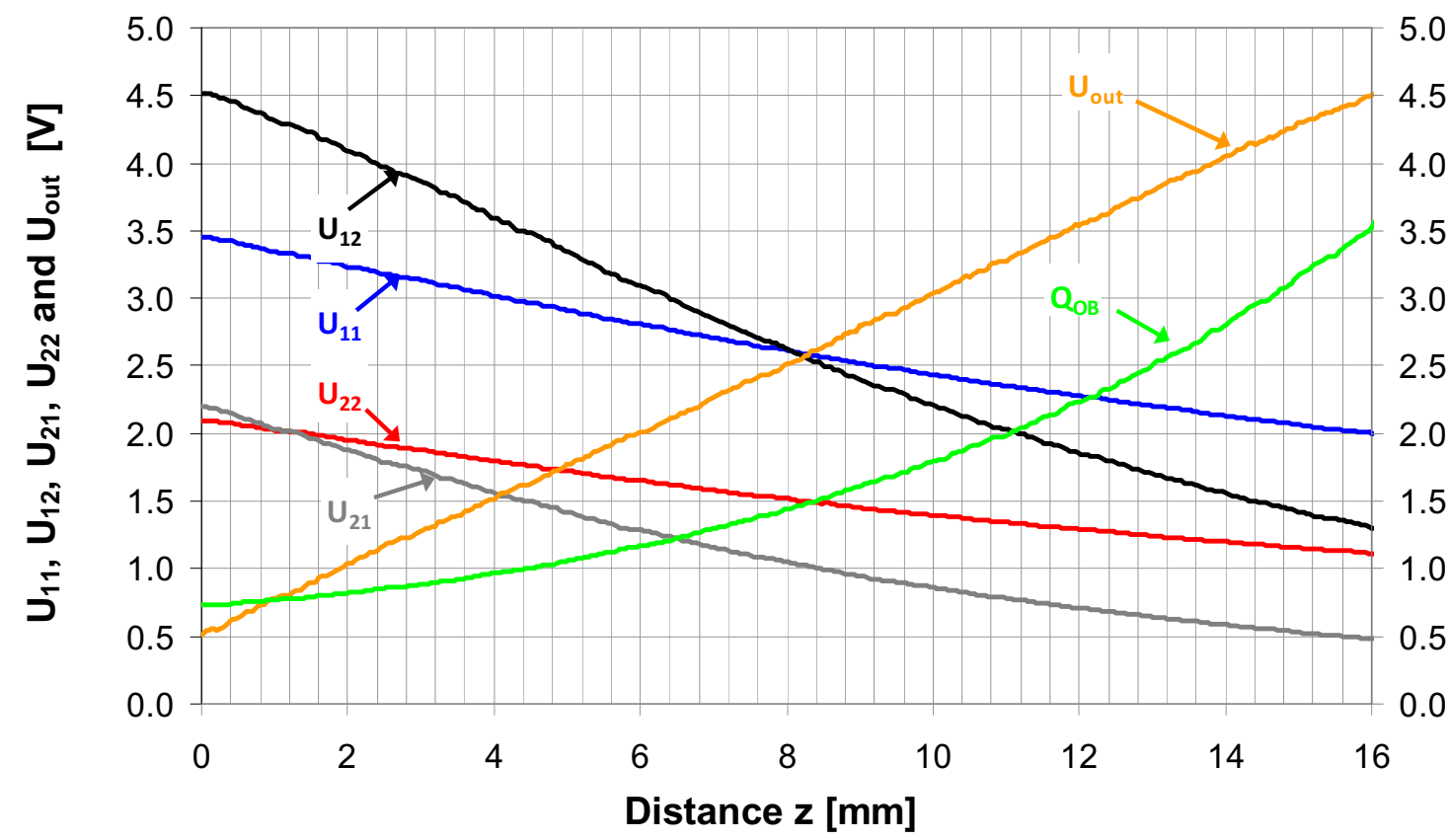

Fig.6. Dependence of voltages $U_{11}, U_{22}, U_{12}, U_{21}$, quotient $Q_{O B}$ and the output voltage of the sensor system $U_{\text {out }}$ in $z$ - axis for one particular realization of the sensor system.

\section{Linearization of the sensor response}

From Figure 6 it is seen that the voltages $U_{11}$, $U_{12}, U_{21}$ and $U_{22}$ of a particular sensor system do not linearly depend on $z$. For linearization, $z$ is calculated as a polynomial of $Q_{O B}$ using the method of least squares. The procedure of finding an approximating polynomial is automated in the assembly line using the
Labview ${ }^{\circledR}$ software. For the sake of simplicity we illustrate the linearization procedure with a second-order polynomial (see Eq. 2.):

$$
z=a_{2} \bullet Q_{O B}{ }^{2}+a_{1} \bullet Q_{O B}{ }^{1}+a_{0} \bullet Q_{O B}{ }^{0}
$$

The coefficients $a_{2}, a_{1}, a_{0}$ are stored in a lowcost microcontroller of the sensor system during the calibration in the assembly line. The distance $z$ is re-calculated into the output voltage $U_{\text {out }}$ of the sensor system using the following equation (3). 
$U_{\text {out }}=f(z)=s \bullet z+o$

Here $s$ is the slope of the curve and $o$ is the offset. Both parameters ( $s$ and o) are included into the parameter set. Finally, Fig. 6 presents the output voltage $U_{\text {out }}$, which is a linear function of $z$.

\section{Measurement procedure}

First, the microcontroller gets the four basic voltage signals by turning first $E_{1}$ on ( $E_{2}$ is off, $U_{11}$ and $U_{12}$ are measured) and then turning $E_{2}$ on ( $E_{1}$ is off; $U_{21}$ and $U_{22}$ are measured). Second, the quotient $Q_{O B}$ is calculated. Third, using $Q_{\mathrm{OB}}$ and the individual calibration coefficients $a_{2}, a_{1}, a_{0}$ the actual distance $z$ to the reflector is determined. This value of $z$ is transformed into the output voltage $U_{\text {out }}$ through equation (3). This voltage is given out via a digital-analog-converter using a PWM output.

\section{Overview of the designed sensor}

The result of the above considerations is a $3 D$ distance sensor system based on the physical principle of optical reflection (see Fig.7.).

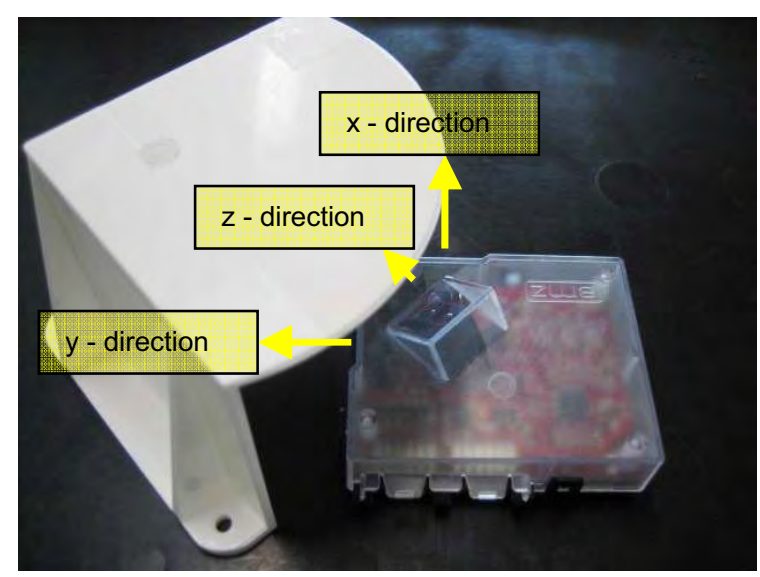

Fig.7. Photograph of the designed 3D - sensor system with the reflector

This sensor system can be currently used in two working modes, which can be selected by a digital input. First is the so-called spinning mode. The optical bridge is not employed there, but the linearization of all three axes works with the same procedure as represented above analog to the optical bridge value $Q_{O B}$ except using the respective analog voltage $U_{\mathrm{ij}}(\mathrm{i}, \mathrm{j}=1$ or 2). In this mode the sensor system can provide distance measurements in all 3 dimensions ranging from $15 \mathrm{~mm}$ to $65 \mathrm{~mm}$ with a measurement frequency of up to $30 \mathrm{~Hz}$.

Second, the sensor system can be operated in the weight mode. The optical bridge configuration is employed. The distance measurement is in $z$-direction only. The measurement range is from $25 \mathrm{~mm}$ to $41 \mathrm{~mm}$ and the typical absolute accuracy is measured to be $\pm 0.3 \mathrm{~mm}$. The measurement frequency in this mode is up to $10 \mathrm{~Hz}$.

\section{Assembly line}

The sensor system described above was developed up to a pre-serial state. On the one hand, the functionality of the sensor system is provided as shown above. On the other hand, an automated assembly line was designed, built up and qualified as well.

At the very beginning the sensor system has to be programmed. Next, the sensor is automatically calibrated in the assembly line (see Fig.8) and gets the individual parameter sets $a_{2}, a_{1}, a_{0}, \mathbf{s}$, o. There are four different sets: three sets for each axis in the spinning mode and one set for the $z$-axis in the weight mode. At least the sensor is measured in all three axes and both modes and evaluated as good or bad part. The entire procedure takes less than 30 seconds for one sensor system. It has to be further optimized for a serial production line.

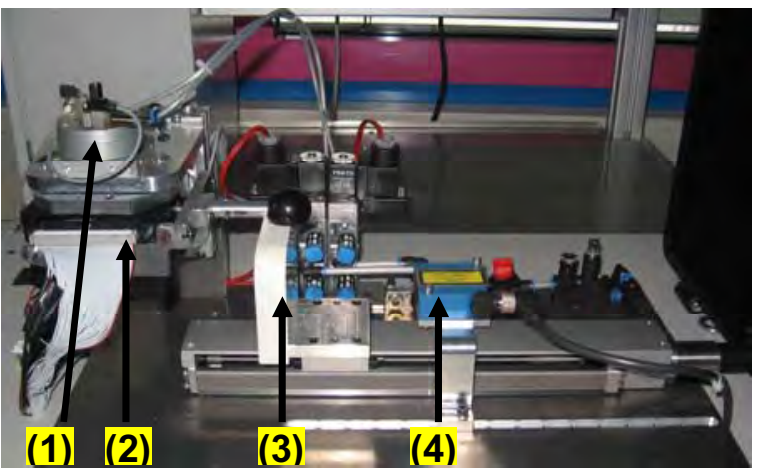

Fig. 8. Photograph of the automated assembly line:

(1) rotating mechanism; (2) sensor fixed and connected by pins; (3) reflector; (4) laser distance sensor.

\section{References}

[1] B. Culshaw, J. Dakin, eds., Optical Fiber Sensors: Systems and Applications Volume 2 (1989), pp. 446-449, Artech House, Norwood.

[2] A. Apelsmeier, B. Schmauss, M. Shamonin, Compensation of parasitic losses in an extrinsic fiber-optic temperature sensor based on intensity measurement, Sens. Actuat. A 173 (2012) 49-54.

[3] G. Murtaza, J.M. Senior, Referencing strategies for intensity modulation of fibre sensors: a review, Optics \& Laser Technology, 25 (1993) 235-245. 
DOI 10.5162/opto2013/o4.1

[4] L. Papula, Mathematische Formelsammlung (2006), Vieweg - Verlag, Wiesbaden; 9. Auflage. 